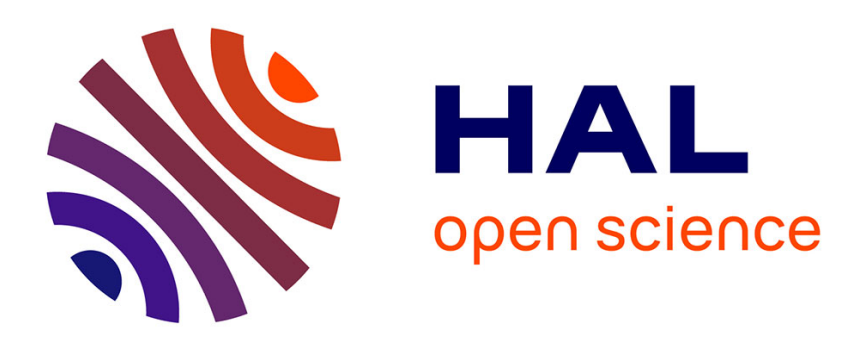

\title{
On multiconsensus of multi-agent systems under aperiodic and asynchronous sampling
}

\author{
Mattia Mattioni
}

\section{To cite this version:}

Mattia Mattioni. On multiconsensus of multi-agent systems under aperiodic and asynchronous sampling. IEEE Control Systems Letters, 2020, 4 (4), pp.839 - 844. 10.1109/LCSYS.2020.2994225 . hal-02638194

\section{HAL Id: hal-02638194 \\ https://hal.science/hal-02638194}

Submitted on 28 May 2020

HAL is a multi-disciplinary open access archive for the deposit and dissemination of scientific research documents, whether they are published or not. The documents may come from teaching and research institutions in France or abroad, or from public or private research centers.
L'archive ouverte pluridisciplinaire HAL, est destinée au dépôt et à la diffusion de documents scientifiques de niveau recherche, publiés ou non, émanant des établissements d'enseignement et de recherche français ou étrangers, des laboratoires publics ou privés. 


\title{
On multiconsensus of multi-agent systems under aperiodic and asynchronous sampling
}

\author{
Mattia Mattioni
}

\begin{abstract}
In this paper, the problem of estimating a suitable bound for ensuring multiconsensus of single integrators under asynchronous and aperiodical sampling is investigated. The estimate relies on a hybrid modeling of the network dynamics with a distributed time-delay acting over the connection. Simulations support the theoretical results.
\end{abstract}

Index Terms-Sampled-data control, Linear systems, Network analysis and control.

\section{INTRODUCTION}

C Onsensus protocols have been widely investigated in the literature in both the continuous and discrete-time contexts (e.g., [1], [2]) as allowing to model and analyse several problems related to different disciplines (e.g., biology, medicine, interstellar navigation and exploration, cybersecurity, etc). It is well known that, at least for continuous-time systems, when scalar integrators are suitably interconnected through a communication graph, the corresponding trajectories cluster and asymptotically converge to common values (that is, multiconsensus [3]); in particular, as recently completely characterized in [4], such clusters and the number of consensus points are related to the topology of the graph through the notion of almost equitable partitions.

Common necessary and sufficient conditions for consensus of continuous-time systems do not apply to the discrete-time context where a further gain (typically referred to as coupling strength) over the interconnection term is needed. In this case, an estimate of such a gain for guaranteeing convergence to consensus is provided by the inverse of the number of components of the network so that, the larger is the network, the smaller is the effect of the interconnection over each agent. However, in these scenarios several works have been developed also covering switching topologies (e.g., [5]) due to their impact on the study, among many, of communication protocols, opinion dynamics or epidemic spread [6], [7].

An appealing case is provided by sampled-data networks where the information among agents (modelled as continuoustime dynamics) is asynchronously exchanged only at sporadic time instants which may be unequally spread over time. Although representative of several practical situations, only a few works are devoted to this scenario, with particular emphasis on (single) consensus-based control for dynamical sampled-data systems (mostly LTI, e.g., [8], [9]) under particular assumptions on the graph topology. However, results on

This work was supported Università degli Studi di Roma La Sapienza (Progetti di Ateneo 2018-Piccoli progetti RP11816436325B63)

Dipartimento di Ingegneria Informatica, Automatica e Gestionale A. Rubert (Università degli Studi di Roma La Sapienza - Via Ariosto 2500185 Rome (Italy) mattia.mattionicuniroma1.it the analysis of the preservation of multiconsensus for multiagent systems under aperiodic and sporadic communication are not available even for single-integrator dynamics despite their study is recognized to be fundamental for the analysis of more complex networks [10], [11]. Moreover, the study of several dynamics (e.g., passive systems, opinion dynamics, routing networks) can be lead to the one of single integrators.

Due to the discrete-time nature of the information exchange, the presence of a suitable coupling gain $\kappa \in \mathbb{R}$ is unavoidable for preserving consensus under sampling. When sampling is periodical and synchronous (with sampling period of length $T>0$ ) the problem can be completely addressed over the sampled-data equivalent model (and hence in the discretetime framework) associated to each agent at all sampling instants $t_{k}=k T$ for $k \geq 0$ so that, whenever the graph is weakly connected, (single)consensus is achieved for all $T>0$ if $\kappa T<\frac{1}{N}$. However, despite not covering multiconsensus, this bound is quite conservative and does not apply for the asynchronous case for which the standalone discrete-time setting is not enough for suitably catching the dynamics of the network.

With no assumption on the graph topology, the purpose of this work is to provide an estimate on the bound of the coupling strength preserving asymptotic convergence of all agents to multiconsensus under sampled-data asynchronous and aperiodic communication and with no knowledge of the sampling time-sequences. The approach we exploit is based on a hybrid modelling of the network dynamics affected by a distributed delay of unknown length over the interconnection term. Accordingly, Lyapunov-Krasovskii arguments are used for computing an estimate of the bound on the coupling gain making the network converge to multiconsensus.

The remaining of the paper is organized as follows: In Section II preliminaries on multiconsensus for continuoustime scalar integrators are given and the problem is formally settled; in III the main result is proved by exploiting a suitable hybrid formulation of the dynamics affected by a perturbating distributed delay; validating simulations are reported in IV while $\mathrm{V}$ concludes the paper.

Notations: $\mathbb{C}^{+}$(resp. $\mathbb{C}^{-}$) denotes the left-hand (resp. righthand) side of the complex plane. $\operatorname{Mat}_{\mathbb{R}}(n, m)$ defines the group of real matrices of dimension $n \times m$ with, for short, $\operatorname{Mat}_{\mathbb{R}}(n)=$ $\operatorname{Mat}_{\mathbb{R}}(n, n)$. Given a matrix $A \in \operatorname{Mat}_{\mathbb{R}}(n), \sigma\{A\} \subset \mathbb{C}$ defines its spectrum. I and $\mathbf{0}$ denote, respectively, the identity and zero matrix of suitable dimensions whereas $\mathbb{1}_{p}$ is the $p$-dimensional vector whose entries are one. For a sorted set of $a_{i} \in \mathbb{R}$ with $i=1, \ldots, n, \operatorname{diag}\left\{a_{1}, \ldots, a_{n}\right\}$ defines a diagonal matrix with $a_{i}$ being the diagonal elements. For $n>1$, given $x_{1}, \ldots, x_{n} \in \mathbb{R}$, we denote $\operatorname{col}\left\{x_{1}, \ldots, x_{n}\right\}:=\left(x_{1} \ldots x_{n}\right)^{\top}$. 


\section{PRELIMINARIES AND PROBLEM STATEMENT}

\section{A. Recalls on graphs and Laplacians}

Consider a digraph (that is unweighted directed graph) $\mathscr{G}=$ $\{\mathscr{V}, \mathscr{E}\}$ with $\mathscr{V}$ being the set of vertices with cardinality $|\mathscr{V}|=$ $N$ and $\mathscr{E} \subseteq \mathscr{V} \times \mathscr{V}$ being the set of edges (i.e., the set of ordered pairs of node). For all pairs of distinct nodes $v_{i}, v_{j} \in \mathscr{V}$ then $\left(v_{i}, v_{j}\right) \in \mathscr{E}$ if there exists an edge from $v_{i}$ to $v_{j}$ or, equivalently, $v_{i}$ is a neighbour of $v_{j}$ for all $i \neq j=1, \ldots, N$. The set of neighbours associated to $v_{i}$ is defined as $\mathscr{N}_{i}:=\{v \in$ $\mathscr{V} /\left\{v_{i}\right\}$ s.t. $\left.\left(v, v_{i}\right) \in \mathscr{E}\right\}$ with cardinality $d_{i}=\left|\mathscr{N}_{i}\right|$ being also referred to as in-degree of $v_{i}$.

Let $\mathscr{R}_{1}, \ldots, \mathscr{R}_{\mu}$ define the reaches of the graph [12], [13]. For each reach $\mathscr{R}_{i}, \mathscr{H}_{i}=\mathscr{R}_{i} / \cup_{j=1, j \neq i}^{\mu} \mathscr{R}_{j}$ with cardinality $h_{i}=\left|\mathscr{H}_{i}\right|$ defines the exclusive part while $\mathscr{C}_{i}=\mathscr{R}_{i} / \mathscr{H}_{i}$ is the corresponding common part whose union defines $\mathscr{C}=\cup_{i=1}^{\mu} C_{i}$ with cardinality $\delta=|\mathscr{C}|$.

The in-degree matrix is defined as $D=\operatorname{diag}\left(d_{1}, \ldots, d_{N}\right) \in$ $\operatorname{Mat}_{\mathbb{R}}(N, N)$ whereas the adjacency matrix is $A=\left\{a_{i j}\right\} \in$ $\operatorname{Mat}_{\mathbb{R}}(N, N)$ with $a_{i i}=0$ and $a_{i j}=1$ if $\left(v_{i}, v_{j}\right) \in \mathscr{E}$ and $a_{i j}=0$ otherwise. The Laplacian of $\mathscr{G}$ is $\mathscr{L}=D-A$ and possesses one eigenvalue $\lambda=0$ with algebraic multiplicity $\mu$ equal to the number of reaches of $\mathscr{G}$ and all other eigenvalues in the left-hand side of the complex plane [13], [14]. As a result, it has been shown in [4] that, after suitably reordering the graphs nodes, the Laplacian $\mathscr{L}$ always admits the triangular form

$$
\mathscr{L}=\left(\begin{array}{ccccc}
\mathscr{L}_{1} & \mathbf{0} & \cdots & \mathbf{0} & \mathbf{0} \\
\mathbf{0} & \mathscr{L}_{2} & \cdots & \mathbf{0} & \mathbf{0} \\
& & \ddots & & \\
\mathbf{0} & \mathbf{0} & \cdots & \mathscr{L}_{\mu} & \mathbf{0} \\
\mathscr{M}_{1} & \mathscr{M}_{2} & \cdots & \mathscr{M}_{\mu} & \mathscr{M}
\end{array}\right)
$$

where $\mathscr{L}_{i} \in \operatorname{Mat}_{\mathbb{R}}\left(h_{i}, h_{i}\right)$ is the Laplacian associated to the strongly connected subgraph induced by the exclusive $\mathscr{H}_{i}$ and $\mathscr{M} \in \operatorname{Mat}_{\mathbb{R}}(\delta, \delta)$ is related the common parts $\mathscr{C}$ of the digraph. As a consequence, each $\mathscr{L}_{i}$ possesses an eigenvalue in $\lambda=0$ with algebraic multiplicity 1 whereas $\sigma(\mathscr{M}) \subset \mathbb{C}^{+}$. By this structure, it is evident that the eigenspace associated to the zero eigenvalue is hence given by $E=\operatorname{span}\left\{w_{1}, \ldots, w_{\mu}\right\}$ with

$$
w_{1}=\left(\begin{array}{c}
\mathbb{1}_{h_{1}} \\
\mathbf{0} \\
\vdots \\
\mathbf{0} \\
\gamma^{1}
\end{array}\right), \quad w_{2}=\left(\begin{array}{c}
\mathbf{0} \\
\mathbb{1}_{h_{2}} \\
\vdots \\
\mathbf{0} \\
\gamma^{2}
\end{array}\right) \quad \ldots w_{\mu}=\left(\begin{array}{c}
\mathbf{0} \\
\mathbf{0} \\
\vdots \\
\mathbb{1}_{h_{\mu}} \\
\gamma^{\mu}
\end{array}\right)
$$

with $\sum_{i=1}^{\mu} \gamma^{i}=\mathbb{1}$ and verifying $\mathscr{L}_{i} \mathbb{1}_{h_{i}}=0$ and $\mathscr{M}_{i} \mathbb{1}_{h_{i}}+\mathscr{M} \gamma^{i}=$ 0 for all $i=1, \ldots, \mu$. Correspondingly, the left eigenvectors associated to the zero eigenvalue of $\mathscr{L}$ are given by

$$
\begin{aligned}
& \tilde{v}_{1}^{\top}=\left(\begin{array}{lllll}
v_{1}^{\top} & \mathbf{0} & \ldots & \mathbf{0} & \mathbf{0}
\end{array}\right), \quad \tilde{v}_{2}^{\top}=\left(\begin{array}{llllll}
\mathbf{0} & v_{2}^{\top} & \ldots & \mathbf{0} & \mathbf{0}
\end{array}\right), \\
& \ldots, \tilde{v}_{\mu}^{\top}=\left(\begin{array}{lllll}
\mathbf{0} & \mathbf{0} & \ldots, & v_{\mu}^{\top} & \mathbf{0}
\end{array}\right)
\end{aligned}
$$

verifying $v_{i}^{\top} \mathscr{L}_{i}=0$ and assumed, without loss of generality, such that $v_{i}^{\top} \mathbb{1}_{h_{i}}=1$ for all $i=1, \ldots, \mu$. Moreover, it can be checked that $v_{i}$ is composed of non-negative coefficients. Moreover, it is clear that if $\mathscr{M}_{i}=\mathbf{0}$ then the reach $\mathscr{R}_{i}$ defines a disconnected component of the graph $\mathscr{G}$.
In the following, we shall denote by $U=\left(\begin{array}{ll}U_{0} & U_{r}\end{array}\right), V=$ $\left(U^{-1}\right)^{\top}=\left(\begin{array}{ll}V_{0} & V_{r}\end{array}\right)$ the full-rank matrices composed of all right and left eigenvectors of the Laplacian (1) with $U_{0}=$ $\left(\begin{array}{lll}w_{1} & \ldots & w_{\mu}\end{array}\right), V_{0}=\left(\begin{array}{lll}\tilde{v}_{1} & \ldots & \tilde{v}_{\mu}\end{array}\right)$ and $U_{r}$ and $V_{r}^{\top}$ such that $V_{r}^{\top} U_{r}=\mathrm{I}$.

\section{B. The continuous-time case}

When associating to each node $v_{i} \in \mathscr{V}$ a dynamical scalarintegrator, the Laplacian $\mathscr{L}$ completely governs the behaviors of the overall network [1], [4]. To this end, consider the Laplacian of the form (1) and each agent dynamics

$$
\dot{x}_{i}(t)=u_{i}(t)
$$

with $x_{i} \in \mathbb{R}$ and $u_{i} \in \mathbb{R}^{n}$ being the coupling term specifying the interaction among all agents through graph Laplacian; namely, setting $\mathbf{u}=-\kappa \mathscr{L} \mathbf{x}$, one gets that the networked system is described by

$$
\dot{\mathbf{x}}(t)=-\kappa \mathscr{L} \mathbf{x}(t)
$$

with $\kappa>0$ being the coupling gain (or strength), $\mathbf{x}_{i}=\operatorname{col}\left\{\left\{x_{j}\right\}_{v_{j} \in \mathscr{H}_{i}}\right\} \in \mathbb{R}^{h_{i}}, \quad \mathbf{u}_{i}=\operatorname{col}\left\{\left\{u_{j}\right\}_{v_{j} \in \mathscr{H}_{i}}\right\} \in \mathbb{R}^{h_{i}}$ for $i=1, \ldots, \mu, \quad \mathbf{x}_{\mathscr{C}}=\operatorname{col}\left\{\left\{x_{j}\right\}_{v_{j} \in \mathscr{C}}\right\} \in \mathbb{R}^{\delta}$, $\mathbf{u}_{\mathscr{C}}=\operatorname{col}\left\{\left\{u_{j}\right\}_{v_{j} \in \mathscr{C}}\right\} \in \mathbb{R}^{\delta}, \mathbf{x}=\operatorname{col}\left\{\mathbf{x}_{1}, \ldots, \mathbf{x}_{\mu}, \mathbf{x}_{\mathscr{C}}\right\} \in \mathbb{R}^{N}$ and $\mathbf{u}=\operatorname{col}\left\{\mathbf{u}_{1}, \ldots, \mathbf{u}_{\mu}, \mathbf{u}_{\mathscr{C}}\right\} \in \mathbb{R}^{N}$.

As proven in [4], for all initial states $\mathbf{x}_{0}=\mathbf{x}(0) \in \mathbb{R}^{N}$ and $\kappa>0$, as $t \rightarrow \infty$, the network reaches multiconsensus that is

$$
\mathbf{x}(t) \rightarrow \mathbf{x}_{c}=\sum_{i=1}^{\mu}\left(\tilde{v}_{i}^{\top} x_{0}\right) w_{i}=\left(\begin{array}{c}
v_{1}^{\top} \mathbf{x}_{1,0} \mathbb{1}_{h_{1}} \\
v_{2}^{\top} \mathbf{x}_{2,0} \mathbb{1}_{h_{2}} \\
\vdots \\
v_{\mu}^{\top} \mathbf{x}_{\mu, 0} \mathbb{1}_{h_{\mu}} \\
\sum_{i=1}^{\mu} v_{i}^{\top} \mathbf{x}_{i, 0} \gamma^{i}
\end{array}\right)
$$

Namely, the network asymptotic behavior is clustered: all agents from the same exclusive subgraph (that is $v_{j} \in \mathscr{H}_{i}$ ) converge asymptotically to the same consensus value; all agents belonging to the common part (that is $v_{j} \in \mathscr{C}$ ) converge to different values which depend on the initial conditions of the agents in the exclusive reaches. In addition, the consensus behavior associated to all agents in $\mathscr{C}$ are clustered as well to further consensus points uniquely defined by the almost equitable partition associated to the graph $\mathscr{G}$. With a slight abuse of notations, considering an almost equitable partition $\pi=\left\{\mathscr{H}_{1}, \ldots, \mathscr{H}_{\mu}, \rho_{\mu+1}, \ldots, \rho_{\varphi}\right\}$ associated to $\mathscr{G}$, one gets:

- $x_{j}(t) \rightarrow v_{i}^{\top} \mathbf{x}_{i, 0}$ for $i=1, \ldots, \mu$ and for all $j \in \mathscr{H}_{i}$;

- $x_{j}(t) \rightarrow c_{i}$ for all $j \in \rho_{i}$ for $i=\mu+1, \ldots, \varphi$.

In other words, the multi-synchronization (or simply synchronization) error defined as $\mathbf{e}=\operatorname{col}\left\{\mathbf{e}_{1}, \ldots, \mathbf{e}_{\mu}, \mathbf{e}_{\mathscr{C}}\right\}=U_{r} V_{r}^{\top} \mathbf{x}$ with, for $i=1, \ldots, \mu$

$$
\mathbf{e}_{i}=\left(I_{h_{1}}-\mathbb{1}_{h_{i}} v_{i}^{\top}\right) \mathbf{x}_{i} \in \mathbb{R}^{h_{i}} \quad \mathbf{e}_{\mathscr{C}}=\mathbf{x}_{\mathscr{C}}-\sum_{i=1}^{h_{i}} \gamma^{i} v_{i}^{\top} \mathbf{x}_{i} \in \mathbb{R}^{\delta}
$$

asymptotically converges to zero. When $\mu=1$ and the graph is undirected, one recovers the well-known results about consensus as the average of the initial states of all agents. 


\section{Problem statement}

So far, we have been considering the case in which all agents can sense the relative positions of the corresponding neighbours continuously in time. However, this case is limiting as the information might be exchanged through digital sensing and providing datas only at sporadic and asynchronous time instants. More in details, let $\Delta_{i}=\left\{t_{0}^{i}, t_{1}^{i}, t_{2}^{i}, \ldots\right\}$ be the unknown sampling sequence associated to the agent $v_{i} \in \mathscr{V}$ with $t_{k+1}^{i}-t_{k}^{i} \in\left[T_{1}, T_{2}\right]$ for all $i=1, \ldots, N$. Then, each agent dynamics (2) reads

$$
\dot{x}_{i}(t)=u_{i}\left(t_{k}^{i}\right), \quad t \in\left[t_{k}^{i}, t_{k+1}^{i}\right)
$$

so that for $t \in\left[t_{k}^{i}, t_{k+1}^{i}\right)$ and $t_{k}^{i}, t_{k+1}^{i} \in \Delta_{i}$ one gets

$$
u_{i}(t)=u_{i}\left(t_{k}^{i}\right)=-\kappa \sum_{j \in \mathscr{N}_{i}}\left(x_{i}\left(t_{k}^{i}\right)-x_{j}\left(t_{k}^{i}\right)\right)
$$

for $i=1, \ldots, N$.

Remark 2.1: The case in which the exchange of information is based on asynchronous data transmission, that is

$$
u_{i}(t)=u_{i}\left(t_{k}^{i}\right)=-\kappa \sum_{j \in \mathscr{N}_{i}}\left(x_{i}\left(t_{k}^{i}\right)-x_{j}\left(t_{k}^{j}\right)\right)
$$

can be developed along the same lines of what is done next.

At this point, although the graph structure stays unchanged (and thus the consensus characterization), asymptotic convergence might be lost in general depending on: the sampling sequence, the degree of asynchronization and the coupling strength. In the following, we estimate an upper bound of the coupling strength $\kappa>0$ so to preserve, in closed loop, asymptotic convergence of all agents to multiconsensus (4) for all possible asynchronous sampling sequences verifying $t_{k+1}^{i}-t_{k}^{i} \in\left[T_{1}, T_{2}\right]$ with $i=1, \ldots, N$ and for a general graph topology. In other words, we seek for a suitable $\kappa^{\star}>0$ such that for all $\kappa \in\left(0, \kappa^{\star}\right)$ the synchronization error (5) asymptotically converges to zero.

Remark 2.2: As typical in the sampled-data context, the velocities of each agent dynamics need to be small with respect to the length of the sampling intervals. This motivates the need of an estimate of the upper bound for the coupling strength $\kappa$ preserving multiconsensus.

Remark 2.3: The problem we address here is different from the one in [15] for consensus-based control of doubleintegrators under aperiodic and asynchronous position measurements. In the aforementioned work, it is assumed that each agent sends its own position at asynchronous and aperiodic time instants over the network whereas here we assume that each agent can sense itself its relative position with respect to the corresponding neighbours at the sampling instants. Secondly, $u_{i}$ in (2) is a continuously varying signal (i.e., $\dot{u}_{i}(t) \neq 0$ for $t \in\left[t_{k}^{i}, t_{k+1}^{i}\right)$ while here we consider the case of piecewise constant coupling terms. Then, in [15], only the case of single-consensus is covered focusing toward control design whereas here the interest relies on the analysis of the effect of sampling onto the network.

We stress that, in the results to come, it is not necessary for the graph Laplacian to be in the form (1).

\section{MAIN RESULT}

\section{A. Hybrid modeling of the network dynamics}

First, by exploiting the standard hybrid model for sampleddata systems [16], we compactly write (6) as

$$
\begin{aligned}
& \left\{\begin{array}{l}
\dot{x}_{i}=u_{i} \\
\dot{u}_{i}=0 \\
\dot{\tau}_{i}=-1
\end{array} \quad\left(x_{i}, u_{i}, \tau_{i}\right) \in C_{i}\right. \\
& \left\{\begin{array}{ll}
x_{i}^{+}=x_{i} \\
u_{i}^{+}=-\kappa \sum_{j \in \mathscr{N}_{i}}\left(x_{i}-x_{j}\right) \\
\tau_{i}^{+} \in\left[T_{1}, T_{2}\right]
\end{array} \quad\left(x_{i}, u_{i}, \tau_{i}\right) \in D_{i}\right.
\end{aligned}
$$

with flow and jump sets defined as $C_{i}=\left\{\left(x_{i}, u_{i}, \tau_{i}\right) \in \mathbb{R} \times\right.$ $\left.\mathbb{R} \times \mathbb{R}: \tau_{i} \in\left[0, T_{2}\right]\right\}, D_{i}=\left\{\left(x_{i}, u_{i}, \tau_{i}\right) \in \mathbb{R} \times \mathbb{R} \times \mathbb{R}: \tau_{i}=0\right\}$ and $\tau_{i}=\tau_{i}(t)=t_{k+1}^{i}-t$ with $t \in\left[t_{k}^{i}, t_{k+1}^{i}\left[\right.\right.$ and $t_{k}^{i}, t_{k+1}^{i} \in \Delta_{i}$ corresponding to a decreasing timer associated to the $i^{\text {th }}$ agent keeping track of the remaining time for the next sampling instant $t_{k+1}^{i}$ to occur for $i=1, \ldots, N$.

Remark 3.1: The hybrid formulation (8) states that discretetime coupling term of the $i^{\text {th }}$ agent is updated whenever new samples are available (that is $t \in \Delta^{i}$ and $\tau_{i}=0$ ) whereas it is kept unchanged otherwise.

Equivalently, setting $\tau=\operatorname{col}\left\{\tau_{1}, \ldots, \tau_{N}\right\}$ the overall network dynamics reads

$$
\begin{cases}\dot{\mathbf{x}}=\mathbf{u}, & \dot{\mathbf{x}}^{+}=\dot{\mathbf{x}} \\ \dot{\mathbf{u}}=\mathbf{0}, & \mathbf{u}^{+}=-\kappa(\mathrm{I}-E(\tau)) \mathscr{L} \mathbf{x}+E(\tau) \mathbf{u} \\ \dot{\tau}=-\mathbb{1}_{N}, & \tau^{+} \in E(\tau) \tau+(\mathrm{I}-E(\tau)) W \\ \dot{r}=-1, & r^{+}=\min \left\{\tau^{+}\right\}\end{cases}
$$

with $W=\left[T_{1}, T_{2}\right] \times \cdots \times\left[T_{1}, T_{2}\right]$, flow and jump sets as

$$
C=\cup_{i=1}^{N} C_{i}, D=\left\{(\mathbf{x}, \mathbf{u}, \tau, r) \in \mathbb{R}^{N} \times \mathbb{R}^{N} \times \mathbb{R}_{\geq 0}^{N}: r=0\right\}
$$

and $E(\tau)=\operatorname{diag}\left\{E_{1}\left(\tau_{1}\right), \ldots, E_{N}\left(\tau_{2}\right)\right\} \in \operatorname{Mat}_{\mathbb{R}}(N, N)$ being a selecting matrix such that $E_{i}\left(\tau_{i}\right)=1$ if $\tau_{i}>0$ and 0 otherwise for $i=1, \ldots, N$.

Remark 3.2: The hybrid model (9) represents the aggregate of all coupled agents (8) with the timer-vector $\tau \in \mathbb{R}^{N}$ being updated according to the inclusion $\tau^{+} \in E(\tau) \tau+(\mathrm{I}-E(\tau)) W$ : only the agents $v_{i} \in \mathscr{V}$ with $\tau_{i}\left(t_{k+1}^{i}\right)=0$ update the corresponding coupling term while re-initializing the corresponding timer. $r(t)>0$ is a further timer keeping track of the next closest jump instant occurring over the network; i.e., $r(t)=t_{k+1}-t$ with $t_{k+1} \in \Delta:=\left\{t_{0}, t_{1}, t_{2}, \ldots\right\}=\cup_{i=1}^{N} \Delta_{i}$ being the union of all sampling sequences and $t \in\left[t_{k}, t_{k+1}\right)$.

Remark 3.3: As $t_{k+1} \in \Delta$ (i.e., $\left.r\left(t_{k+1}\right)=0\right), E(\tau)$ selects all agents $v_{j} \in \mathscr{V}$ for which $\tau_{j}\left(t_{k+1}\right)>0$ (that is $t_{k} \notin \Delta_{j}$ ); namely, $u_{j}\left(t_{k+1}\right)=u_{k}\left(t_{k}\right)$ if $\tau_{j}\left(t_{k+1}\right)>0$. Similarly, $E\left(\tau\left(t_{k}\right)\right) \ldots E\left(\tau\left(t_{0}\right)\right)$ is of the same form with coefficients on the diagonal being 0 if the corresponding agent has sensed new measures at least once over $t \in\left[t_{0}, t_{k}\right)$ and 1 otherwise.

Because all agents' sampling periods are upper bounded by $T_{2} \geq 0$, when $t \geq T_{2}$ all agents have updated the corresponding coupling term at least once and the graph corresponding to $t \in\left[0, T_{2}\right]$ is jointly connected [5]. In this respect, picking $t_{\bar{k}}>$ $T_{2}$ with $\bar{k} \leq N$ and $t_{\bar{k}} \in \Delta$, one gets $E\left(\tau\left(t_{\bar{k}}\right)\right) \ldots E\left(\tau\left(t_{0}\right)\right)=0$. As a straightforward consequence, letting $\hat{N} \leq N$ be such that 
$t_{k}-t_{k-\hat{N}} \geq T_{2}$ and $t_{k} \in \Delta$, one has $E\left(\tau\left(t_{k}\right)\right) \ldots E\left(\tau\left(t_{k-\hat{N}}\right)\right)=0$. Accordingly, for all $t_{k} \in \Delta$, the coupling law is given by $u\left(t_{k}\right)=-\kappa \mathscr{L} \mathbf{x}\left(t_{k}\right)+E\left(\tau\left(t_{k}\right)\right) p(k)$ with $p \in \mathbb{R}^{n}$ being a perturbing time-delay term of the form

$$
\begin{aligned}
p(k)= & \kappa \mathscr{L} \mathbf{x}\left(t_{k}\right) \\
& -\kappa \sum_{\ell=k-\hat{N}}^{k-1} E\left(\tau\left(t_{k}\right)\right) \ldots E\left(\tau\left(t_{\ell+1}\right)\right)\left(\mathrm{I}-E\left(\tau\left(t_{\ell}\right)\right) \mathscr{L} \mathbf{x}\left(t_{\ell}\right) .\right.
\end{aligned}
$$

From this rewriting it is evident that the overall network system is intrinsically affected by a distributed delay of unknown length $\hat{N} \leq N$.

Remark 3.4: The perturbation term $p$ is modelled as a discrete variable with zero derivative over the flowing time as part of the piecewise constant coupling law.

\section{B. Consensus under aperiodic and asynchronous sampling}

As a consequence of the hybrid model (9), the analysis must unavoidably take into account both the hybrid nature of the system and the effect of the distributed delay which is intrinsically induced by the sampled-data communication.

For consensus to be achieved, one needs to suitably estimate the upper bound $\kappa^{\star}>0$ for the coupling gain $\kappa>0$ such that $\mathbf{e}(t) \rightarrow 0$ as $t \rightarrow \infty$ with synchronization error given in (5). In the hybrid domain, this corresponds to prove asymptotic stability of the set

$$
\mathscr{A}=\left\{(\mathbf{x}, \mathbf{u}) \in \mathbb{R}^{N} \times \mathbb{R}^{N}: \mathbf{e}=0\right\} \times\left\{\left[0, T_{2}\right]\right\}^{N} \times\left[0, T_{2}\right]
$$

for the hybrid model (9) with in particular $\mathbf{e} \equiv 0$, if and only if $\mathbf{u}=0$ and $\mathbf{x} \in \operatorname{ker}\left\{V_{r}^{\top}\right\}=\operatorname{span}\left\{w_{1}, \ldots, w_{\mu}\right\}$.

For the sake of simplicity and as typical in this context [10], introduce the coordinate transformation ${ }^{1}$

$$
\left(\begin{array}{c}
\tilde{\mathbf{x}} \\
\tilde{\mathbf{u}}
\end{array}\right)=\left(\mathrm{I} \otimes V^{\top}\right)\left(\begin{array}{l}
\mathbf{x} \\
\mathbf{u}
\end{array}\right), \Lambda=V^{\top} \mathscr{L} U=\left(\begin{array}{cc}
\mathbf{0}_{\mu \times \mu} & \mathbf{0} \\
\mathbf{0} & \Lambda_{r}
\end{array}\right)
$$

with $\Lambda_{r}=\operatorname{diag}\left\{\lambda_{1}, \ldots, \lambda_{N-\mu}\right\}$ and $\lambda_{i} \in \sigma(\mathscr{L})-\{0\}$ for all $i=1, \ldots, N-\mu$. Also, setting $\tilde{\mathbf{x}}=\operatorname{col}\left\{\tilde{\mathbf{x}}_{o}, \tilde{\mathbf{x}}_{r}\right\} \in \mathbb{R}^{\mu} \times \mathbb{R}^{N-\mu}$ and $\tilde{\mathbf{u}}=\operatorname{col}\left\{\tilde{\mathbf{u}}_{o}, \tilde{\mathbf{u}}_{r}\right\} \in \mathbb{R}^{\mu} \times \mathbb{R}^{N-\mu}$, one gets

$$
\begin{aligned}
& \left\{\begin{array}{l}
\dot{\tilde{\mathbf{x}}}_{o}=\tilde{\mathbf{u}}_{o}, \quad \dot{\tilde{\mathbf{u}}}_{o}=\mathbf{0} \\
\dot{\tilde{\mathbf{x}}}_{r}=\tilde{\mathbf{u}}_{r}, \quad \dot{\tilde{\mathbf{u}}}_{r}=\mathbf{0} \\
\dot{\tau}=-\mathbb{1}_{N}, \quad \dot{r}=-1
\end{array}\right. \\
& \left\{\begin{array}{l}
\tilde{\mathbf{x}}_{o}^{+}=\tilde{\mathbf{x}}_{o}, \quad \tilde{\mathbf{u}}_{o}^{+}=V_{0}^{\top} E(\tau) p \\
\tilde{\mathbf{x}}_{r}^{+}=\tilde{\mathbf{x}}_{r}, \quad \tilde{\mathbf{u}}_{r}^{+}=-\kappa \Lambda_{r} \mathbf{x}_{r}+V_{r}^{\top} E(\tau) p \\
\tau^{+} \in E(\tau) \tau+(\mathrm{I}-E(\tau)) W, \quad r^{+}=\min \left\{\tau^{+}\right\}
\end{array}\right.
\end{aligned}
$$

with

$$
\begin{aligned}
p(k) & =\kappa U_{r} \Lambda_{r} \tilde{\mathbf{x}}_{r}\left(t_{k}\right) \\
& -\kappa \sum_{\ell=k-\hat{N}}^{k-1} E\left(\tau\left(t_{k}\right)\right) \ldots E\left(\tau\left(t_{\ell+1}\right)\right)\left(\mathrm{I}-E\left(\tau\left(t_{\ell}\right)\right) U_{r} \Lambda_{r} \tilde{\mathbf{x}}_{r}\left(t_{\ell}\right) .\right.
\end{aligned}
$$

Denoting $\zeta=\operatorname{col}\left\{\tilde{\mathbf{x}}_{o}, \tilde{\mathbf{x}}_{r}, \tilde{\mathbf{u}}_{o}, \tilde{\mathbf{u}}_{r}, \tau, r\right\}$ and

$$
f(\zeta)=\left(\begin{array}{c}
\tilde{\mathbf{u}} \\
0 \\
-\mathbb{1} \\
-1
\end{array}\right), \quad G(\zeta)=\left(\begin{array}{c}
\tilde{\mathbf{x}} \\
-\kappa \Lambda \tilde{\mathbf{x}}+V^{\top} E(\tau) p \\
E(\tau) \tau+(\mathrm{I}-E(\tau)) W \\
\min \left\{\tau^{+}\right\}
\end{array}\right)
$$

${ }^{1}$ With no loss of generality, we assume $\sigma(\mathscr{L}) \in \mathbb{R}$. the dynamics (9) compactly reads as

$$
\dot{\zeta}=f(\zeta), \quad \zeta \in C ; \quad \zeta^{+} \in G(\zeta), \quad \zeta \in D
$$

Because $\mathbf{e}=U_{r} V_{r}^{\top} \mathbf{x}=U_{r} \mathbf{x}_{r}$ and $U_{r}$ is full-column rank, one gets that $\mathbf{e} \equiv 0$ if and only if $\tilde{\mathbf{x}}_{r} \equiv 0$ and $\tilde{\mathbf{u}}_{r} \equiv 0$.

Remark 3.5: In those coordinates the network possesses a further upper triangular structure: $\tilde{\mathbf{x}}_{O}$ and $\tilde{\mathbf{u}}_{o}$ are not influenced by $\tilde{\mathbf{x}}_{r}$ and $\tilde{\mathbf{u}}_{r}$.

Theorem 3.1: Consider the sampled-data agents (6) in closed loop with the control protocol (7) and interconnected according to the graph defined by the Laplacian (1). Multiconsensus is achieved for all sampling sequences $\Delta_{k}^{i}=\left\{t_{0}^{i}, \ldots, t_{k}^{i}, \ldots\right\}$ with $i=1, \ldots, N$ and $T_{2} \geq T_{1}>0$, if the coupling gain $\kappa>0$ verifies

$$
\kappa<\kappa^{\star}=\frac{2 T_{1} q_{m}}{T_{2}\left(\hat{\alpha}+T_{2}\right) q_{M}^{2}}
$$

with $q_{m}$ and $q_{M}$ being the smallest and largest nonzero eigenvalues of $\mathscr{L}$ and an arbitrary $\hat{\alpha}>0$.

Proof. Denoting $\zeta_{t_{k}}=\zeta\left(t_{k+\theta}, k+\theta\right)$ with $\theta \in[-\hat{N}, 0)$, consider the Lyapunov-Krasovskii candidate

$$
\mathscr{W}\left(\zeta(t), \zeta_{t_{k}}\right)=V(\zeta(t))+\gamma W\left(\zeta\left(t_{k}\right)\right)
$$

with, for $\alpha>0, t \in\left[t_{k}, t_{k+1}\right)$ and $t_{k+1}>t_{k} \in \Delta$

$$
\begin{aligned}
& W\left(\zeta\left(t_{k}\right)\right)=\sum_{\ell=k-\hat{N}}^{k} \sum_{j=\ell}^{k}\left\|V_{r}^{\top} \mathbf{E}\left(\tau\left(t_{\left[\ell, t_{k}\right]}\right)\right) U_{r} \Lambda_{r} \tilde{\mathbf{x}}_{r}\left(t_{j}\right)\right\|^{2} \\
& V(\zeta)=\left(\begin{array}{ll}
\tilde{\mathbf{x}}_{r}^{\top} & \tilde{\mathbf{u}}_{r}^{\top}
\end{array}\right) e^{A_{r}^{\top} r} P e^{A_{r} r}\left(\begin{array}{c}
\tilde{\mathbf{x}}_{r} \\
\tilde{\mathbf{u}}_{r}
\end{array}\right), \quad P=\left(\begin{array}{cc}
\mathrm{I} & 0 \\
0 & \alpha I
\end{array}\right) \succ 0 \\
& A_{r}=\left(\begin{array}{cc}
\mathbf{0}_{N-\mu \times N-\mu} & \mathrm{I}_{N-\mu} \\
\mathbf{0}_{N-\mu \times N-\mu} & \mathbf{0}_{N-\mu \times N-\mu}
\end{array}\right) \\
& \mathbf{E}\left(\tau\left(t_{\left[j, t_{k}\right]}\right)\right)= \begin{cases}\mathbf{0} & \ell=k \\
E\left(\tau\left(t_{k}\right)\right) \ldots\left(E ( \tau ( t _ { \ell + 1 } ) ) \left(\mathrm{I}-E\left(\tau\left(t_{\ell}\right)\right),\right.\right. & \ell<k .\end{cases}
\end{aligned}
$$

By non-singularity of $e^{A_{r} r}$ and positive-definiteness of $P$ and $V V^{\top}$, it is verified that the above functional is indeed a Lyapunov-Krasovskii candidate being zero if and only if $\zeta \in \mathscr{A}$ (that is $e=U_{r} \mathbf{x}_{r} \equiv 0$ ). It is easily checked that during flows (i.e., for $\left.t \in\left[t_{k}, t_{k+1}\right)\right), \dot{\mathscr{W}}\left(\zeta_{t}\right)=0$. On the other side, during jumps (i.e., at $t=t_{k+1}$ ) and setting

$$
g(\zeta)=\left(\tilde{\mathbf{x}}^{\top} \quad\left(-\kappa \Lambda \tilde{\mathbf{x}}+V^{\top} E(\tau) p\right)^{\top} \quad w_{\tau}^{\top} \quad w_{r}\right)^{\top} \in G(\zeta)
$$

with $w_{\tau} \in\left[T_{1}, T_{2}\right] \times \cdots \times\left[T_{1}, T_{2}\right]$ and $w_{r} \in\left[T_{1}, T_{2}\right]$, one has

$$
\begin{aligned}
\Delta V(\zeta):= & V(g(\zeta))-V(\zeta) \\
= & -\kappa \tilde{\mathbf{x}}_{r}^{\top}\left(w_{r}\left(\Lambda_{r}+\Lambda_{r}^{\top}\right)-\kappa\left(\alpha+w_{r}^{2}\right) \Lambda_{r}^{\top} \Lambda_{r}\right) \tilde{\mathbf{x}}_{r} \\
& +2 p^{\top} E(\tau) V_{r}\left(w_{r} \mathrm{I}-\kappa\left(w_{r}^{2}+\alpha\right) \Lambda_{r}\right) \tilde{\mathbf{x}}_{r} \\
& +\left(w_{r}^{2}+\alpha\right) p^{\top} E(\tau) V_{r} V_{r}^{\top} E(\tau) p-\alpha\left\|\tilde{\mathbf{u}}_{r}\right\|^{2}
\end{aligned}
$$

with by definition $-\left(\Lambda_{r}+\Lambda_{r}^{\top}\right) \preceq-2 q_{m} I \prec 0$. Invoking the Young inequality, one gets that for all $d_{1}>0$

$$
\begin{aligned}
& 2 p^{\top} E(\tau) V_{r}\left(w_{r} \mathrm{I}-\kappa\left(w_{r}^{2}+\alpha\right) \Lambda_{r}\right) \tilde{\mathbf{x}}_{r} \\
\leq & \frac{1}{d_{1}} p^{\top} E(\tau) V_{r} V_{r}^{\top} E(\tau) p+d_{1} \tilde{\mathbf{x}}_{r}^{\top} Q_{1}^{\top} Q_{1} \mathbf{x}_{r}
\end{aligned}
$$


with $Q_{1}=w_{r} \mathrm{I}-\kappa\left(w_{r}^{2}+\alpha\right) \Lambda_{r}$. Accordingly, one gets

$$
\begin{aligned}
\Delta V(\zeta) \leq & -\kappa \tilde{\mathbf{x}}_{r}^{\top}\left(w_{r} q_{m} \mathrm{I}-\kappa\left(\alpha+w_{r}^{2}\right) \Lambda_{r}^{\top} \Lambda_{r}-\frac{d_{1}}{\kappa} Q_{1}^{\top} Q_{1}\right) \tilde{\mathbf{x}}_{r} \\
& +\left(w_{r}^{2}+\alpha+\frac{1}{d_{1}}\right) p^{\top} E(\tau) V_{r} V_{r}^{\top} E(\tau) p-\alpha\left\|\tilde{\mathbf{u}}_{r}\right\|^{2} .
\end{aligned}
$$

For the functional component, as $E(\tau)(\mathrm{I}-E(\tau)) \equiv 0$, we get $W\left(\zeta_{t_{k}}^{+}\right)-W\left(\zeta_{t_{k}}\right)=-W_{0}\left(\zeta_{t_{k+1}}\right) \leq 0$ with $W_{0}\left(\zeta_{t_{k+1}}\right)=$ $\sum_{j=k-\hat{N}}^{k+1} \| V_{r}^{\top} \mathbf{E}\left(\tau\left(t_{j, k+1}\right) U_{r} \Lambda_{r} \tilde{\mathbf{x}}_{r}\left(t_{j}\right) \|^{2}\right.$ so that one has for $p=$ $p(k+1)$

$$
\begin{aligned}
p^{\top} E(\tau) V_{r} V_{r}^{\top} E(\tau) p & \leq \kappa^{2} \| \sum_{j=k-\hat{N}}^{k+1} V_{r}^{\top} \mathbf{E}\left(\tau\left(t_{j, k+1}\right) U_{r} \Lambda_{r} \tilde{\mathbf{x}}_{r}\left(t_{j}\right) \|^{2}\right. \\
& \leq \kappa^{2} \hat{N} W_{0}\left(\zeta_{t_{k+1}}\right) .
\end{aligned}
$$

Accordingly, one gets that

$$
\begin{aligned}
\Delta \mathscr{W}\left(\zeta, \zeta_{t_{k}}\right):= & \left.\mathscr{W}(g(\zeta)), \zeta_{t_{k+1}}\right)-\mathscr{W}\left(\zeta, \zeta_{t_{k}}\right) \\
\leq & -\kappa \tilde{\mathbf{x}}_{r}^{\top}\left(2 w_{r} q_{m} \mathrm{I}-\kappa\left(\alpha+w_{r}^{2}\right) \Lambda_{r}^{\top} \Lambda_{r}-\frac{d_{1}}{\kappa} Q_{1}^{\top} Q_{1}\right) \tilde{\mathbf{x}}_{r} \\
& +\left(\gamma-\kappa^{2} \hat{N}\left(w_{r}^{2}+\alpha+\frac{1}{d_{1}}\right)\right) W_{0}\left(\zeta_{t_{k+1}}\right)-\alpha \mathbf{u}_{r}^{\top} \mathbf{u}_{r} \\
\leq & -\kappa\left(2 q_{m} T_{1}-\kappa\left(\alpha+T_{2}^{2}\right) q_{M}^{2}-\frac{d_{1}}{\kappa}\left\|Q_{1}^{\top} Q_{1}\right\|\right)\left\|\tilde{\mathbf{x}}_{r}\right\|^{2} \\
& +\left(\gamma-\kappa^{2} \hat{N}\left(w_{r}^{2}+\alpha+\frac{1}{d_{1}}\right)\right) W_{0}\left(\zeta_{t_{k+1}}\right)-\alpha\left\|\mathbf{u}_{r}\right\|^{2}
\end{aligned}
$$

with $q_{M}=\max \{\sigma\{\mathscr{L}\}\}$. Hence, $\Delta \mathscr{W}\left(\zeta, \zeta_{t_{k}}\right)<0$ if $\gamma>$ $\kappa^{2} \hat{N}\left(w_{r}^{2}+\alpha+\frac{1}{d_{1}}\right)$ and $q_{m} T_{1}-\kappa\left(\alpha+T_{2}^{2}\right) q_{M}^{2}>0$ for all $\alpha>0$ and for $d_{1}>0$ small enough concluding that $\mathscr{A}$ is asymptotically stable for (9) if the coupling strength verifies (13) with $\alpha=T_{2} \hat{\alpha}$. The remaining part of the proof on the analysis of the flow interval is omitted as follows the lines of standard results [16].

Remark 3.6: From (13), the convergence rate to multiconsensus is inversely proportional on the term $\frac{T_{1}}{T_{2}}$ which can be hence interpreted as the degree of synchronization: as $T_{2}>>T_{1}$ convergence is slow whereas faster as $T_{2} \rightarrow T_{1}$.

Remark 3.7: In Theorem 3.1 the constant $\hat{\alpha}>0$ can be chosen arbitrarily small as it is needed to guarantee $\mathbf{u}(t) \rightarrow 0$ as $t \rightarrow \infty$. In that case, whenever $T_{2} \rightarrow T_{1} \rightarrow 0$ and $\hat{\alpha} \rightarrow 0$, one gets $\kappa^{\star} \rightarrow \infty$ so recovering the continuous-time result stating that consensus is achieved for all $\kappa>0$.

Remark 3.8: $\kappa^{\star}$ is directly proportional to $q_{m}$, the smallest non-zero eigenvalue of $\mathscr{L}$ as in (1), that reflects the properties of the least connected component $\mathscr{H}_{i}$ (with $\left.i=1, \ldots, \mu\right)$ of $\mathscr{G}$.

Remark 3.9: The above result is comparable with the one in [5] for discrete-time systems under time-varying topology and weakly connected graph (that is in case of single consensus). Indeed, fixing $\kappa \leq \frac{1}{T_{2} N}$ one has that the network converges to consensus as $t_{k} \geq T_{2}$ one has that for all $t \in\left[t_{k}, t_{k}+T_{2}\right.$ [ the explored graph is jointly connected so that consensus is achieved. However, this result does not take into account the sampled-data nature of the agents.

Remark 3.10: By the structure of the Laplacian (1), Theorem 3.1 covers the case of disconnected components as well.

Remark 3.11: Neither knowledge of the left and right eigenvectors of the Laplacian (1) nor of the underlined block
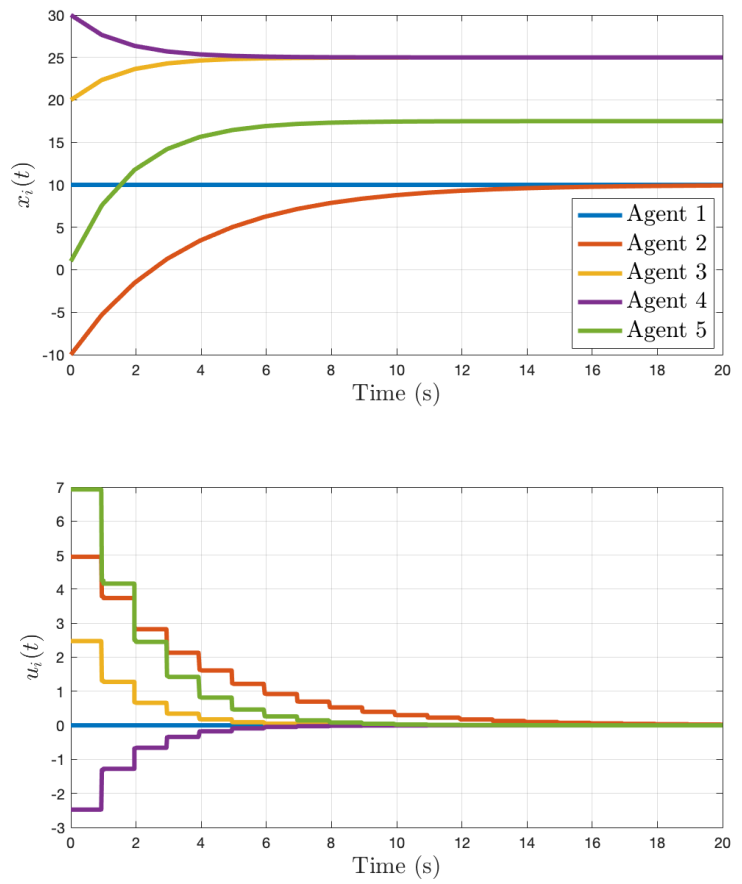

Fig. 1: $T_{1}=T_{2}=1, \kappa=0.2475$

diagonal structure are necessary for estimating the upper bound of the coupling gain.

Remark 3.12: As typical in communication networks, one might have $\Delta^{i} \cap \Delta^{j}=\emptyset$ for $i \neq j$ and $i, j=1, \ldots, N$ [7]; namely, the sampling instants of all agents are different $\left(t_{k}^{i} \neq t_{k}^{j}\right.$ for all $k \geq 0$ and $i \neq j)$. Accordingly, for all $t_{k} \in \Delta$ and $k \geq 0$, one gets $u_{j}\left(t_{k}\right)=u_{j}\left(t_{k-1}\right)$ for $j \neq i$ whereas $u_{i}\left(t_{k}\right)=-\kappa l_{i} \mathbf{x}$ with $l_{i}$ corresponding to the $i^{\text {th }}$ row of the Laplacian (1). In such a case, one can substitute $q_{m}$ and $q_{M}$ in (13) with $d_{m}$ and $d_{M}$ being, respectively, the smallest and largest elements on the main diagonal of the Laplacian $\mathscr{L}$ (i.e., the smallest and largest in-degrees among all nodes).

Remark 3.13: The functional exploited in the proof of Theorem 3.1 is reminiscent of the continuous-time one in [17] for asymptotic stability under delays and sampling.

\section{Simulations AND COMPARISONS}

Consider the case of a network governed by the Laplacian

$$
\mathscr{L}=\left(\begin{array}{ccccc}
0 & 0 & 0 & 0 & 0 \\
-1 & 1 & 0 & 0 & 0 \\
0 & 0 & 1 & -1 & 0 \\
0 & 0 & -1 & 1 & 0 \\
-1 & 0 & -1 & 0 & 2
\end{array}\right)
$$

composed by 5 agents and three potential consensus points: two for the exclusive reaches $\mathscr{H}_{1}=\left\{v_{1}, v_{2}\right\}$ and $\mathscr{H}_{2}=\left\{v_{3}, v_{4}\right\}$ and one for the common $\mathscr{C}=\left\{v_{5}\right\}$. In Figures 1 to 3 ,

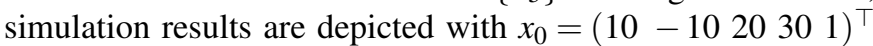
and $\kappa=0.99 \kappa^{\star}$ with $\kappa^{\star}$ as (13) with $\hat{\alpha}=1$ and several sampling scenarios. In Figure 1 the case of synchronous and periodic sampling is reported. In Figure 2 the degree of 

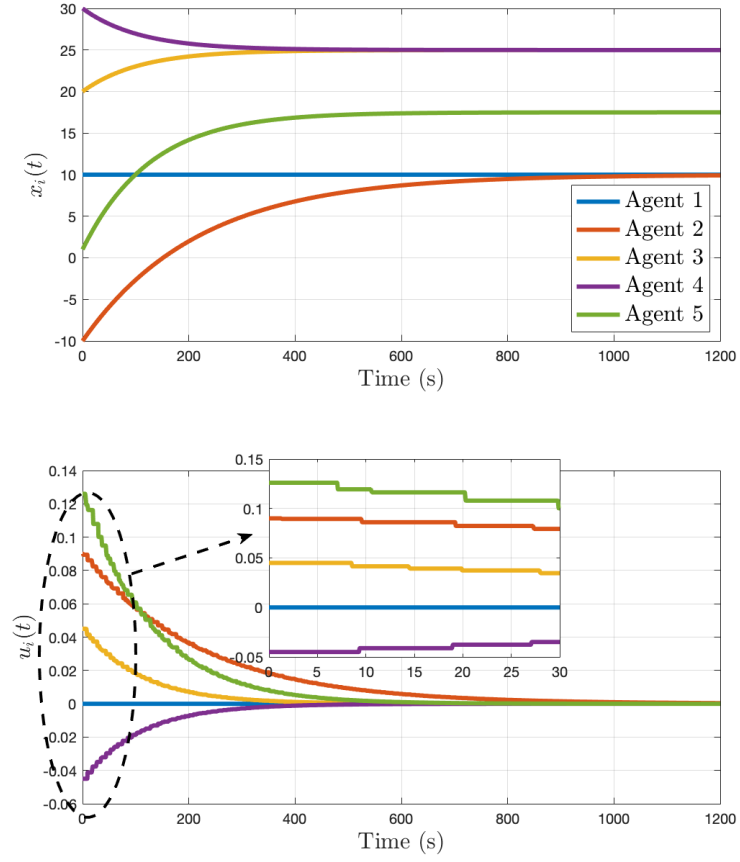

Fig. $2: T_{1}=1, T_{2}=10, \kappa=0.0045$

asynchronization is higher so that convergence to multiconsensus is much slower than in Figure 3 where the average of the sampling intervals is higher but with smaller deviation (Remark 3.6). Further tests show that the estimate (13) is close to the actual bound for high degree of asynchronism.

\section{CONCLUSIONS}

We have provided estimates on the coupling strength ensuring multiconsensus of single-integrators under asynchronous and aperiodic sampling and general graph topology. The analysis is based on a hybrid formulation of the dynamics also embedding a distributed delay due to asynchronism. Future works concern switching topologies and the case of agents with general nonlinear dynamics.

\section{ACKNOWLEDGMENT}

The author wishes to thank: Prof. Salvatore Monaco for mentoring him through this discipline and, in particular, multiagent systems; Dr. Dorothée Normand-Cyrot for the constant support and teaching; the anonymous Reviewers for the significant suggestions and comments.

\section{REFERENCES}

[1] R. Olfati-Saber, J. A. Fax, and R. M. Murray, "Consensus and cooperation in networked multi-agent systems," Proceedings of the IEEE, vol. 95, no. 1, pp. 215-233, 2007.

[2] W. Ren and R. W. Beard, Distributed consensus in multi-vehicle cooperative control. Springer.

[3] M. Frasca, L. V. Gambuzza, A. Buscarino, and L. Fortuna, Synchronization in Networks of Nonlinear Circuits: Essential Topics with

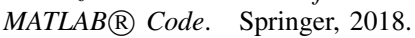
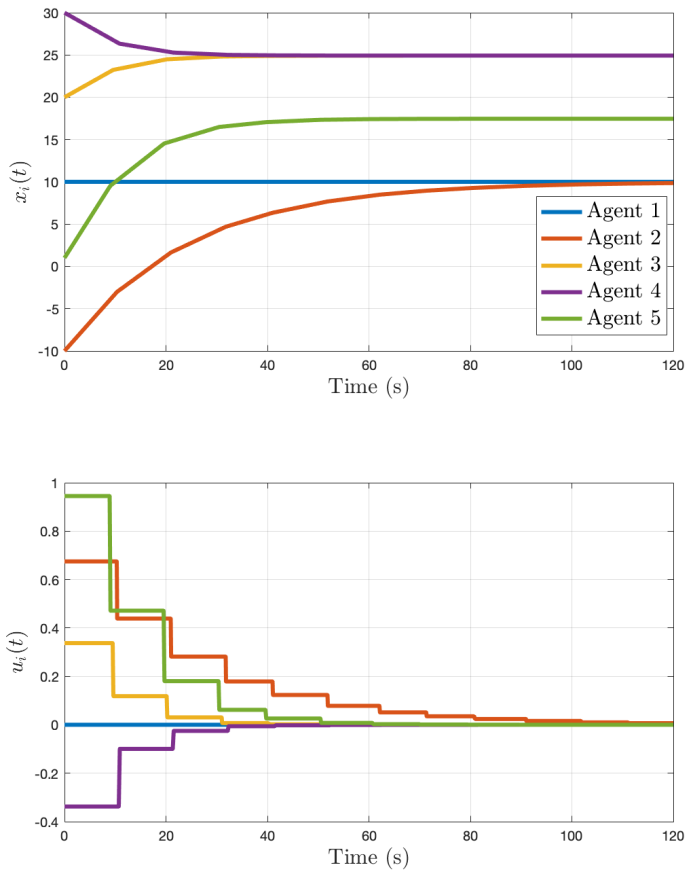

Fig. 3: $T_{1}=9, T_{2}=11, \kappa=0.038$

[4] S. Monaco and L. Ricciardi Celsi, "On multi-consensus and almost equitable graph partitions," Automatica, vol. 103, pp. 53-61, 2019.

[5] A. Jadbabaie, J. Lin, and A. S. Morse, "Coordination of groups of mobile autonomous agents using nearest neighbor rules," Departmental Papers (ESE), p. 29, 2003.

[6] A. V. Proskurnikov, C. Ravazzi, and F. Dabbene, "Dynamics and structure of social networks from a systems and control viewpoint: A survey of roberto tempo's contributions," Online Social Networks and Media, 2018.

[7] A. Giuseppi and A. Pietrabissa, "Wardrop equilibrium in discrete-time selfish routing with time-varying bounded delays," IEEE Transactions on Automatic Control, pp. 1-1, 2020.

[8] Y. Wu, H. Su, P. Shi, Z. Shu, and Z.-G. Wu, "Consensus of multiagent systems using aperiodic sampled-data control," IEEE transactions on cybernetics, vol. 46, no. 9, pp. 2132-2143, 2015.

[9] Y. Hayashida, L. Hetel, T. Oguchi, and J.-P. Richard, "Stability of sampled-data systems with uncertain time-varying delays and its application to consensus control of multi-agent systems," IFAC-PapersOnLine, vol. 50, no. 1, pp. 1257-1262, 2017.

[10] A. Isidori, Lectures in feedback design for multivariable systems. Springer, vol. 3 .

[11] E. Panteley and A. Loría, "Synchronization and dynamic consensus of heterogeneous networked systems," IEEE Transactions on Automatic Control, vol. 62, no. 8, pp. 3758-3773, 2017.

[12] C. Godsil and G. F. Royle, Algebraic graph theory. Springer Science \& Business Media, 2013, vol. 207.

[13] J. S. Caughman and J. Veerman, "Kernels of directed graph Laplacians," the electronic journal of combinatorics, vol. 13, no. 1, p. 39, 2006.

[14] R. Agaev and P. Chebotarev, "On the spectra of nonsymmetric Laplacian matrices," Linear Algebra and its Applications, vol. 399, pp. 157-168, 2005.

[15] T. Ménard, E. Moulay, P. Coirault, and M. Defoort, "Observer-based consensus for second-order multi-agent systems with arbitrary asynchronous and aperiodic sampling periods," Automatica, vol. 99, pp. 237 $-245,2019$.

[16] R. Goebel, R. G. Sanfelice, and A. R. Teel, Hybrid Dynamical Systems: modeling, stability, and robustness. Princeton University Press, 2012.

[17] F. Mazenc, M. Malisoff, and T. N. Dinh, "Robustness of nonlinear systems with respect to delay and sampling of the controls," Automatica, vol. 49, no. 6, pp. 1925 - 1931, 2013. 